\title{
Estimulación vagal en el tratamiento de la epilepsia
}

\author{
J. Iriarte ${ }^{\text {a,c }}$, E. Urrestarazu ${ }^{\text {a }}$ D. Lázaro ${ }^{\text {a }}$, E. Schlumberger ${ }^{\text {b }}$
}

VAGAL STIMULATION IN THE TREATMENT OF EPILEPSY

\begin{abstract}
Summary. Introduction. The vagal nerve stimulation is a new technique for the treatment of drug-resistant epilepsies. Development. In 1997, it was approved in United States by the FDA to be used in adults with refractory focal epilepsies not candidates for epilepsy surgery. Its mechanism of action is unknown. The results in the controlled studies indicated a decrease of $30-50 \%$ in the seizure frequency in around $50 \%$ of the patients. Although more experience is needed to corroborate these results, it seems reasonable as a treatment for patients with difficult epilepsies, especially when the response to the antiepileptic drugs is poor or they are producing secondary effects, and the resection of the focus is not possible. [REV NEUROL 2002; 34: 511-8]
\end{abstract}

Key words. Drug-resistant epilepsy. Vagal stimulation.

\section{INTRODUCCIÓN}

El final del sigloxxha supuesto un avance importante en el tratamientodelas epilepsias. Sin embargo, apesardelaintroducción de nuevos fármacos antiepilépticos y del progreso en la cirugía de la epilepsia, sigue existiendoun grupoimportante de pacientes con crisis frecuentes que no son susceptibles de tratamientoquirúrgico. Alrededorde un 25\% de los pacientes noestán controladososufren efectos secundarios importantes por la medicación antiepiléptica. Se calcula que, en Estados Unidos, los pacientes tratados adecuadamente sin controlde las crisis son unos 150.000-300.000 [1-3]. Extrapolando estos datos, en España serían unos 20.000-40.000 pacientes. Alrededor de un 20-40\% de estas personas pueden ser susceptibles de tratamiento quirúrgico. Por tanto, dado el importante númerode pacientes con epilepsia todavíamal controlada, se siguen planteandootras técnicas terapéuticas. Entreéstas destacan la dieta cetógena y el estimulador vagal.

El intento de controlar las crisis epilépticas mediante estimulación cerebral noes nuevo. Laestimulación cerebelosa pronto se abandonó por su ineficacia $[1,4]$. Otro campo de investigación es la estimulación profunda, especialmente del núcleo centromediano del tálamo. Los resultados preliminares parecen positivos, pero deben considerarse con precaución [5-7].

Encuanto ala estimulación del nervio vago, a partirde los años treinta comenzó a descubrirse que provocaba cambios en elEEG, pero este fenómenorealmente no se estudió y se aplicó hasta más tarde, cuando se planteóla posibilidad de su efecto antiepiléptico [8,9].El primerestimulador vagal se colocó en 1988 [10]. Desde entonces, lainvestigaciónenestecampohasidointensa[11]. Nueve años después, laFDA(Food andDrug Administration)estadounidense aprobó su utilización como tratamiento añadido en adultos y adolescentes, porencima de 12 años de edad, que sufren epilepsia

Recibido: 27.09.01. Acepta dotrasrevisiónexternasinmodificaciones:05.11.01. ${ }^{a}$ Unidad de Epilepsia. ${ }^{b}$ Unidad de Neurología Infantil. Clínica Universitaria. Universidad de Navarra. ${ }^{c}$ Servicio de Neurofisiología Clínica. Hospital Virgen del Camino. Pamplona, España.

Correspondencia: Dr. Jorge Iriarte. Servicio de Neurofisiología Clínica. Clínica Universitaria. Universidad de Navarra. Pío XII, s/n. E-31080 Pamplona, Navarra.E-mail:jiriarte@unav.es

Presentado en el I Congreso de la Liga Española contra la Epilepsia, celebrado en Bilbao del 14 al 17 de noviembre de 2001.

C 2001, REVISTADENEUROLOGÍA rebelde al tratamiento médico, con crisis de inicio focal y no susceptibles de beneficiomediante cirugía [12]. Actualmente, laestimulación vagal cuenta con abundante experiencia tanto en modelos animales como en pacientes. Se han publicado más de 100 artículos sobre su mecanismo de acción, estudios experimentales, aplicación en pacientes y efectos secundarios [13]. Hasta la fecha se ha utilizado en más de 7.000 pacientes en unos 350 centros de epilepsia de todoelmundo. Enel presente trabajo se exponen datos sobre aspectos técnicos del sistema, hipótesis actuales sobre su mecanismo de acción, eficacia y efectos secundarios.

\section{ASPECTOS TÉCNICOS}

La colocación del estimulador vagal (generador y electrodo) es relativamente sencilla $[14,15]$. Se puede realizar con anestesia general o con anestesia cervical. La cirugía suele durar unas dos horas y las complicaciones quirúrgicas son mínimas. El generador habitualmente utilizado, NCP 100, pesa $55 \mathrm{~g}$ y tiene un grosor de 13,2 mm. Últimamente se ha introducido en el mercado el NCP 101, más ligero (38 g) y delgado (10,3 mm), y con una duración más prolongada. Se coloca subcutáneamente en el área infraclavicular (Fig. 1). El cable de conexión entre ambos se introduce por un túnel realizado durante la cirugía a través del tejido subcutáneo. El electrodobipolar de platino se coloca sobre el nervio vago izquierdo, con un sistema de dos hélices espiroidales que abrazan el nervio (Fig. 2).

Una vez colocado el estimulador se mide laimpedancia del electrodo, preferiblemente en quirófano o, en caso contrario, en la habitación del enfermo o la consulta del médico. A continuación se programa el generador utilizando un mando transcutáneo. La intensidad máxima, el intervalo de incremento de intensidad, el período de ascenso y el tiempo de estímulo y descanso varían según la experiencia de los centros y deben adecuarse a la tolerancia y efectos en cada paciente. Habitualmente, los parámetros iniciales son: intensidad de $0,25 \mathrm{~mA}$ y frecuencia de $30 \mathrm{~Hz}$, con estímulo durante $30 \mathrm{~s}$ cada 5 min. Después, cada dos semanas, se aumenta la intensidad hasta alcanzar 2-3 mA, siempre que no se produzcan efectos indeseables.

El paciente o un familiar disponen de un imán con el que, en casodenecesidad, puedenactivarelgeneradoraplicándolosobre el mismo durante 1-3 s. Si se mantiene apoyado sobre el generador se produce un bloqueo de la estimulación. Del primer 
modo, el mismo paciente procura frenar o abortar una crisis que cree inminente. Del segundo, se detiene el funcionamiento del estimulador en el momento en que se producen molestias importantes al paciente. Es preciso que el paciente, la familia y el cuidador entiendan bien el sistema y las señales de alarma, y así puedan actuar oportunamente en caso de crisis o de efectos secundarios.

El generador tiene una duración limitada. Dependiendo del nivel de la estimulación y de la resistencia del electrodo, puede oscilar entre cuatro y ocho años (el NCP 100), o entre ocho y 12 (elNCP 101). Pasado este tiempo es necesario proceder al recambio del generador mediante una nueva cirugía, esta vez sin modificarelelectrodo.Elelectrodo, una vezfijadoen la cirugíainicial, queda adherido al nervio vago por una rica fibrosis local. Puede extraerse quirúrgicamente sin problemas [16], pero la práctica habitual es mantenerlo en su lugar aunque deje de utilizarse.

Al no interferir con otros tratamientos, la colocación del estimulador vagal prácticamente no tiene contraindicaciones absolutas. En pacientes con arritmias cardíacas, alteraciones autonómicas o trastornos de la coagulación, su indicación debe meditarse bien por los riesgos que puede conllevar la cirugía de colocación del dispositivo o la propia estimulación vagal. Recientemente, el fabricante ha notificado la contraindicación de tratamientos con onda corta y ultrasonidos en estos pacientes, tanto si el generador está activado o no. No se incluye en la realización de ecografías. El motivo es la producción de calor local en elárea del generador y del electrodo, que podría producir lesiones importantes en tejidos blandos, nervios y, lo que podría ser más grave, vasos sanguíneos [17].

Tras colocar el estimulador vagal es posible realizar resonancias magnéticas si se requiere por algún motivo. Inicialmente se manifestó cautela ante esta prueba por la posibilidad de mover el electrodo o el generador, o por la producción de calor local. El único problema parece ser la desactivación del generador durante la aplicación del campo magnético, lo que resulta posible evitar mediante la colocación del generador en sentido paralelo al eje del cuerpo [18].

\section{MECANISMO DE ACCIÓN}

Aunque el mecanismo de acción no se conoce, se piensa que los estímulos eléctricos podrían inhibir la sincronización en distintos núcleos que deben descargaral unísono paraquela actividad eléctrica cerebral normal se convierta en una crisis epiléptica. El acceso a la estimulación del cerebro a través del nervio vago se haplanteado como una posibilidad por la amplia difusión de sus conexiones, por ser técnicamente asequible y por no llevar fibras que produzcan dolor. El principal lugar donde el nervio deja aferencias es el núcleo del tracto solitario. Otras fibras proyectan a la formación reticular pontina, el núcleo ambiguo, elnúcleoespinaldeltrigémino, elárea postrema, el núcleodorsal del vago y el núcleocunneatus. Desde estos núcleos, las aferencias continúan hacia el sistema límbico, el hipotálamo, el tálamo y la corteza insular. El núcleo del tracto solitario establece conexiones con otros núcleos del troncoencéfalo, ellocuscoeruleus, los núcleos del rafe y la formación reticular. Estos núcleos, a su vez, pueden hacer variar directa o indirectamente la excitabilidad cortical y la actividad del sistema límbico. La estimulación puede alterar los circuitos en diferentes niveles, pero no se ha probado cuál de ellos justifica una acción antiepiléptica. Sin embargo, aún está por dilucidarse cómo la acción en estos circuitos influye sobre el control de crisis epilépticas. El punto final parece ser un aumento de la hiperpolarización lenta en neuronas corticales [19]. Un dato interesante es el aumento de la expresión de FOS (proteína nuclear indicativa de incremento de actividad neuronal) en la amígdala posterior, el cíngulo, la corteza retroesplenial, los núcleos arcuato y ventromedial del tálamo, los núcleos del vago, ellocus coeruleusy los núcleos cocleares [20]. En ratas se ha demostrado que lesiones dellocus coeruleus, tanto agudas como crónicas, disminuyen la eficacia del estimulador vagal en el control de crisis [21].

Existen múltiples datos que apoyan su eficacia como antiepiléptico. Al aplicarlo en animales se ha observado su utilidad en varios de los tests habituales para determinar el poder antiepiléptico y anticonvulsionante. Los trabajos clásicos de Bailey y Zanchetti demostraron cambios en elEEG, con aumento de ondas a frecuencias altas, aumento del umbral en el modelo de estricnina tópica y disminución de puntas intercríticas; sin embargo, estos datos no se aplicaron hasta que fueron confirmados más recientemente por otros investigadores, también con modelos de estricnina y pentiletilentrazol en perros y ratas, y umbral de electroshock en ratones [22-26]. Rutecki, en el modelo de estricnina tópica en ratas, describe tanto sincronización como desincronización en distintas frecuencias en elEEG, y la disminución de puntas intercríticas [27]. En gatos a los cuales se ha producidokindling eléctrico en la amígdala, el uso del estimulador vagal retrasa o evita la producción de crisis espontáneas [28]. Produce, además, cambios en la actividad eléctrica normal, aumentandoel sueño REMy la densidad de ondas PGO. También altera el comportamiento, produciendo aumento del apetito, bostezos y fenómenos como miosis, parpadeo, salivación, contracciones abdominales, desviación de la mirada hacia arriba y movimientos bruscos de la cabeza. Algunos estudios refieren cambios en la frecuencia de base en elEEG, dependiendo de la intensidad de la estimulación. Utilizando electrodos intracraneales se han encontrado cambios en la frecuencia de las descargas epileptiformes [29]. Algunos estudios no han confirmado cambios en el EEG, ni a simple vista ni en análisis de frecuencias [30-33]. Incluso, para algunos autores, no evita la producción de nuevas crisis [34].

Otro modo de estudiar la actuación del estimulador vagal consiste en detectar cambios inducidos en el sistema nervioso central. Se ha descrito la existencia de respuestas evocadas en la corteza cerebral, el hipocampo, el tálamo y el cerebelo [33].En los potenciales evocados se ha observadoun aumento delatencia de la conducción periférica y central de los PESS, sin cambios en los PEV y PEA, lo que sugiere cambios también en el sistema periférico [35,36]. Otros cambios funcionales corticales se han estudiado mediante SPECT y PET. Los resultados no son homogéneos. Estudios mediante PET con agua indicaron un aumento en el flujo sanguíneo cerebral durante las fases de estimulación del nervio vago izquierdo, siendo significativo especialmente en el tálamo derecho, la corteza temporal posterior bilateral, el putamenizquierdo y el cerebeloizquierdo inferior [37,38]. Henry et al encontraron un aumento de flujo en el hipotálamo, el tálamo, la corteza insular, el giro poscentral, el área inferior del cerebelo y la cara rostral del bulbo, y una disminución de modo bilateral en el hipocampo, la amígdala y elárea posterior del giro cingular [39]. En un estudio posterior encontraron correlación entre el aumento delflujotalámico bilateral y una reducción significativa del número de crisis [40], lo que sugería que el efecto antiepiléptico podríarelacionarse con un aumento de actividad sinápticaen 


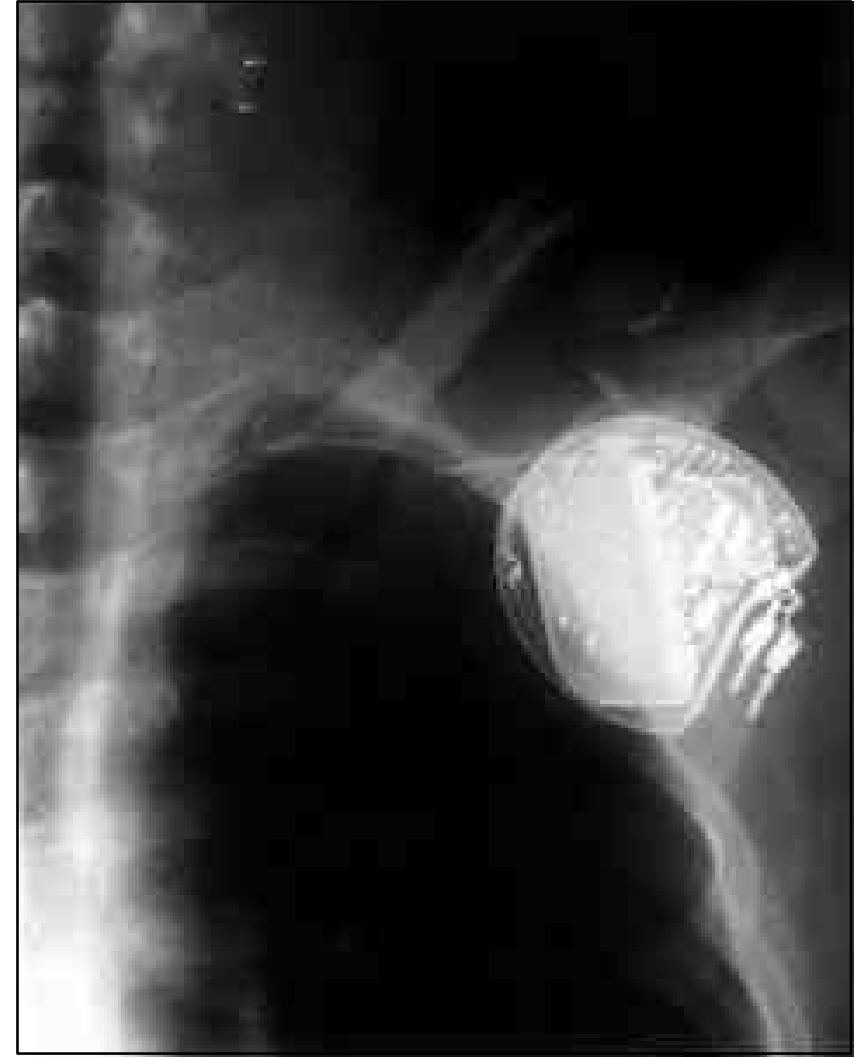

Figura 1. Rx donde se observa el generador en posición infraclavicular.

el tálamo. Llama la atención que, en otros estudios, se haya encontrado una hipoperfusión [41]. Otros estudios con SPECT con tecnecio-99 destacan la hipoperfusión del tálamo homolateral a la estimulación, ya en la fase inicial de uso del estimulador, sin encontrar correlación entre el cambio de la frecuencia de crisis y los hallazgos en SPECT [42].

También se han aportado datos del análisis del líquido cefalorraquídeo(LCR). Se han estudiado los cambios producidos en la concentración de distintos metabolitos y aminoácidos [43]. Se determinóunacorrelación lineal entrelareducción de crisis ylas concentraciones de asparragina, fenilalanina, fosfoetanolamina, alanina y triptófano. La fosfoetanolamina aumentóen los pacientes con buena respuesta clínica y disminuyó en los que no se beneficiaron del tratamiento. Los niveles de GABA estaban aumentados, tantoen los pacientes que mejoraron comoen aquellos en los que no hubo cambio en la frecuencia de crisis. También se objetivó un descenso en la concentración de glutamato.

Los estudios en animales no han servido únicamente para investigar el mecanismo de acción y valorar la actividad antiepiléptica. También han resultadoútiles para determinar la máxima intensidad y parámetros de estimulación sin producir daño químico o eléctrico [44]. Esto es importante porque los parámetros del estímulo constituyen una variable fundamental relacionada con el control de las crisis y con otros aspectos fisiológicos [45].

\section{EFICACIA EN EL CONTROL DE CRISIS Estudios realizados}

Los estudios previos a la aprobación del uso de estimulador vagal como técnica habitual estándar incluyeron cinco estudios

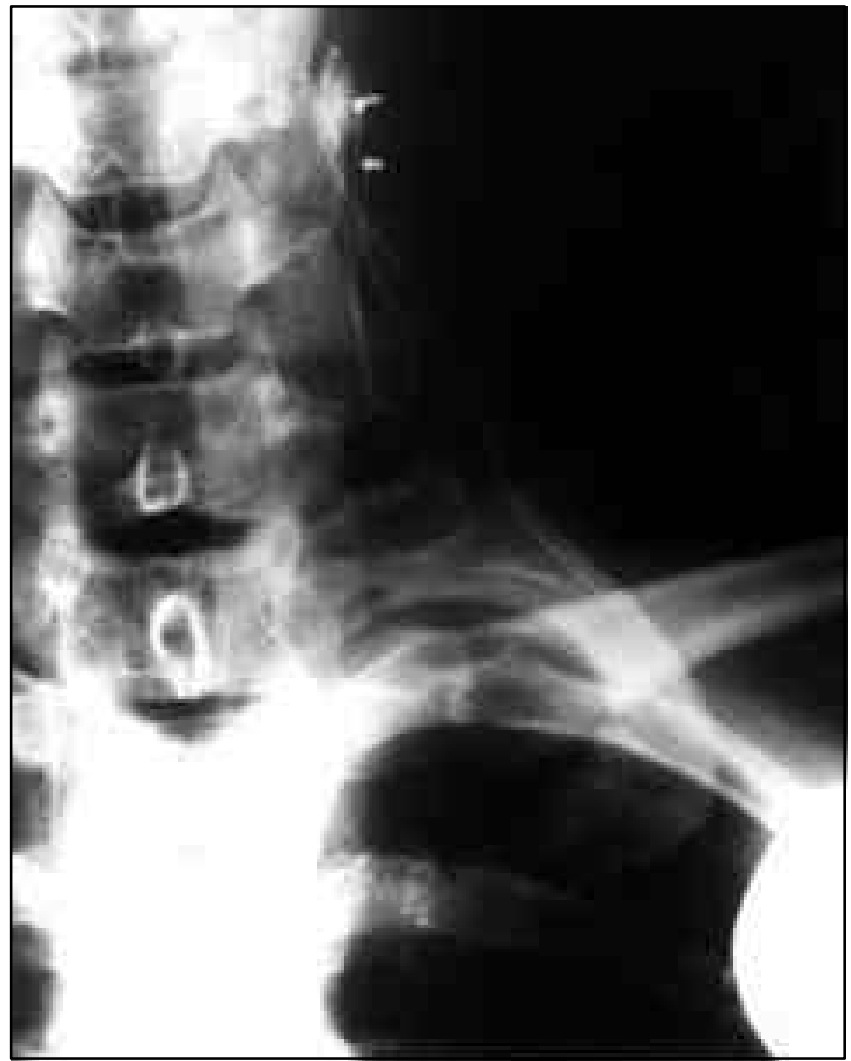

Figura 2. Rx cervical. Electrodo.

controlados (Tabla I). Estos estudios se centraron en eficacia, efectos secundarios y comparación de eficacia de intensidad baja y alta. Se formó un grupo para el estudio de la estimulación del nervio vago en epilepsia. Los datos aportados por este y otros grupos fueron evaluados por un subcomité de la Academia Americana de Neurología y condujeron a la redacción de los informes sobre el estimulador vagal [46,47]. Los resultados fueron favorables, considerando la resistencia de las crisis de los pacientes incluidos en los estudios. Además, en los últimos años se han ido sucediendo comunicaciones de diversos grupos en los que se expresa su experiencia en cuanto a eficacia y efectos secundarios se refiere.

\section{Eficacia a corto plazo}

Los resultados se muestran en la tabla II. Los estudios EO1 y EO2 calcularon una reducción de crisis en la fase aguda del $28 \%$, con un $37 \%$ de pacientes que presentaban una disminución superior al 50\% [3]. El estudio EO3 fue un estudio aleatorizado multicéntrico, doble ciego, en 17 centros de Estados Unidos, Canadá y Europa. Se incluyó a pacientes con epilepsias focales farmacorresistentes; inicialmente se publicaron los resultados de los primeros 67 pacientes [48], y después, del grupo completo [49]. Los sujetos con el estímulo intenso (1,5 mA, 30 $\mathrm{Hz}, 30$ s cada $5 \mathrm{~min}$ ) presentaron a las 14 semanas una reducción en el número de crisis del $31 \%$; el grupo de pacientes con estímulo inferior $(1,25 \mathrm{~mA}, 1 \mathrm{~Hz}, 30 \mathrm{~s}$ cada $90 \mathrm{~min})$ tuvo una reducción de crisis del $11 \%$. En el conjunto de 114 pacientes incluidos finalmente en el estudio, se obtuvo una reducción de un $25 \%$ en el número de crisis en tres meses, y de un $32 \%$ en el seguimiento abierto durante un año. Al dejar de funcionar el genera- 
dor, la frecuencia de crisis se mantuvo estable durante dos semanas y, después, volvió a la frecuencia habitual [49]. Los estudios EO4 y EO5 incluyeron a otros 124 y 196 pacientes, respectivamente. El EO4 era un estudio abierto como tratamiento compasivo en pacientes con diagnóstico de epilepsia parcial o generalizada. Los datos son propiedad de la compañía y hasta el momento se han publicado los resultados en los pacientes con epilepsia generalizada. En estos sujetos, la media de reducción de crisis fue del 46\% [50], y 11 enfermos tuvieron una reducción superior al 50\%. Respondieron mejor aquellos de mayor edad en el momento del inicio de las crisis y aquellos con crisis más frecuentes. El EO5 fue el segundo estudio aleatorizado, ciego, en pacientes entre 13 y 60 años de edad, con crisis parciales mal controladas mediante fármacos antiepilépticos [51]. Se establecieron dos grupos al azar: uno, con estimulación alta, y otro, con estimulación baja. La disminución de crisis fue significativamente mayor en el grupo de estimulación alta $(28 \%$ frente a $15 \%, \mathrm{p}<0,04)$. El porcentaje de pacientes con una reducción del $50 \%$ de crisis fue similar en ambos grupos, si bien el de pacientes con una reducción de175\% fue significativamente mayor entre los de estimulación alta.

\section{Eficacia a medioplazo}

A los pacientes incluidos en los estudios controlados se los siguió de modo abierto a lo largo de períodos más prolongados (12-36 meses). Los resultados son bastante similares (Tabla III). El seguimiento durante 25 meses de los pacientes incluidos en los estudios EO1 y EO2 [3] mostró unos resultados incluso mejores que en la fase aguda, con una reducción de crisis del $46 \%$, y un $35 \%$ de pacientes con una mejoría superior al $50 \%$. El primer grupo de pacientes del EO3, cuya totalidad recibió estimulación alta, fue revisado a los 18 meses [52]. La disminución de crisis con respecto al nivel de base era del $52 \%$, y el $44 \%$ de los pacientes tenía una reducción mayor del $50 \%$ en el número de crisis. En el grupo con estimulación alta se comprobó que las crisis con generalización secundaria ocurrían en un número menor de pacientes que en el grupo que había recibido estimulación baja. Posteriormente se publicaron los datos del grupo total de pacientes, siendo los resultados ligeramente inferiores [53], con un beneficio aproximado del $32 \%$ en frecuencia de crisis y un $31 \%$ de pacientes con una mejoría superior al $50 \%$. Pacientes procedentes delEO5 se incluyeron en un estudio denominado XE5. Pasado un año con la estimulación máxima, la reducción en el número de crisis era del $45 \%$, y se encontró un $35 \%$ de pacientes con una reducción igual o superior al 50\% [54]. El análisis de los cambios clínicos y de los cambios en los parámetros de estimulación llevó a la conclusión de que los cambios en la estimulación no fueron los factores primordiales para obtener mayor eficacia, aunque algunos pacientes se beneficiaran de una menor duración del períodosin estímulo [55]. Tras un seguimiento de 15 meses en los mismos pacientes del XE5, el39\% alcanzaba una reducción del $50 \%$; de ellos, el $21 \%$ presentó una reducción superior al $75 \%$, y dos estaban libres de crisis [56].

El seguimiento abierto de los pacientes incluidos en los estudios EO1 a EO5 dio unos valores similares de eficacia en el control de crisis: una reducción de crisis media de $35 \%$ en el primer año, $44,3 \%$ al cabo del segundo año, y $44,1 \%$ pasados tres años [57].

\section{Experiencia en niños}

En niños, la experiencia es menor, aunque parece igualmente optimista [58-61]. Hornig et al, en un estudio sobre 19 niños, obtuvo que $10(53 \%)$ mejoraron en la frecuencia de crisis más de un $50 \%$, y seis de ellos (32\%) presentaron una reducción en el número de crisis superior al $90 \%$ [62]. Lundgren et al citan que de seis niños con síndrome de Lennox-Gastaut, cinco presentaron una reducción de crisis superior al 90\% [63]. Otros autores confirman esta eficacia y señalan una mejoría ligeramente inferior, con seis pacientes de 13 con una mejoría superior al $50 \%$ y un único paciente sin mejoría significativa [64]. La selección de los pacientes adolescentes de los estudios EO1 a EO5 [65] aporta resultados similares a los de los adultos; destaca que, tras doce meses de seguimiento, un $17 \%$ de pacientes mejoran más del $90 \%$ en el número de crisis. Recientemente, datos de 38 pacientes con edades comprendidas entre 11 meses y 16 años indican una eficacia y seguridad similares a las de los adultos [66], ampliando el espectro de las crisis que mejoran a las crisis tónicas y a la ausencia de las mismas. También pacientes con hamartomas hipotalámicos y crisis gelásticas mejoraron en la frecuencia de crisis (tres de los seis pacientes) o en el comportamiento autista (cuatro pacientes) [67].

\section{Otros estudios}

Los últimos años han sido pródigos en la publicación de resultados de la estimulación del nervio vago en grupos particulares. La colocación de los primeros estimuladores vagales aportaba ya un beneficio claro; de los cuatro primeros pacientes implantados, dos estuvieron libres de crisis durante unos meses, uno mejoró un 40\%, y el otro permanecióigual [10]. Años después, Ben-Menachem et al aportaron una casuística de 64 pacientes con una media de seguimiento de 20 meses [68]. Incluyeron tanto a pacientes con epilepsia parcial como a pacientes con epilepsia generalizadaidiopática o síndrome de Lennox-Gastaut. E145\% de los pacientes presentó una reducción de más del $50 \%$ de las crisis, más frecuente en los pacientes con Lennox-Gastaut (cinco de ocho). Los efectos colaterales fueron leves y poco frecuentes. Amar et al, en una serie de 18 pacientes con crisis parciales complejas o secundariamente generalizadas, encontraron grandes diferencias entre estimulación alta y baja; a estimulación elevada obtuvieron una reducción de crisis del $80 \%$ en tres meses. Respondieron muy positivamente seis de siete pacientes, e incluso uno de ellos estuvo libre de crisis durante el año y medio de seguimiento [69]. Otros autores confirman la 
Tabla II. Resultados a corto plazo.

\begin{tabular}{lcc|cc}
\hline & $\begin{array}{c}\text { \% Reducción } \\
\text { de crisis }\end{array}$ & $\begin{array}{c}\text { Diferencias } \\
\text { entre intensidad } \\
\text { alta y baja }\end{array}$ & $\begin{array}{c}\text { \% de pacientes } \\
\text { con reducción } \\
\text { del 50\% }\end{array}$ & $\begin{array}{c}\text { Diferencias } \\
\text { entre intensidad } \\
\text { alta y baja }\end{array}$ \\
\hline Uthman 1993 [3] & 28 & - & 37 & - \\
\hline Ben-M enachen 1994[48] & $31^{\mathrm{a}}$ & $\mathrm{p}<0,04$ & 39 & No significativo \\
\hline VNSSG 1995 [49] & 25 & $\mathrm{p}<0,01$ & 31 & $\mathrm{p}<0,02$ \\
\hline Handforth 1998 [51] & $28^{\mathrm{a}}$ & $\mathrm{p}<0,04$ & 23 & No significativo \\
\hline a Con parámetros de estimulación alta.
\end{tabular}

Tabla III. Resultados a medio plazo.

\begin{tabular}{lccc}
\hline & $\begin{array}{c}\text { Seguimiento } \\
\text { (meses) }\end{array}$ & $\begin{array}{c}\text { \% Reducción } \\
\text { de crisis }\end{array}$ & $\begin{array}{c}\text { \% Pacientes con } \\
\text { reducción de 50\% }\end{array}$ \\
\hline Uthman 1993[3] & 25 & 46 & 35 \\
\hline George 1994[52] & 18 & 52 & 44 \\
\hline Salinsky 1996[53] & 12 & 32 & 31 \\
\hline Amar 1995[56] & 15 & 37 & 39 \\
\hline Morris 1999 [57] & 12 & 35 & 43 \\
\hline Morris 1999 [57] & 24 & 44 & 43 \\
\hline Morris 1999 [57] & 36 & 44 & 35 \\
\hline De Giorgio 2000 [54] & 12 & 45 & 37 \\
\hline
\end{tabular}

buena respuesta en pacientes con epilepsia bitemporal [70] y epilepsia secundariamente generalizada o parcial [71-73]. En adultos de edad avanzada, los resultados también son prometedores [74].

Aunque no con tanta frecuencia, existen grupos cuya experiencia con respecto al control de crisis por la estimulación vagal no es tan positiva. Parker et al, por ejemplo, en su serie de 15 niños, tuvieron que extraer un estimulador por una infección; en el resto, la reducción de crisis fue únicamente del $17 \%$, con mejoría significativa sólo en cuatro pacientes $(27 \%)$ [75]. Koutroumanidis et al también comunicaron un marcado empeoramiento en el número de crisis y aparición de crisis nuevas [76].

\section{OTRAS ACCIONES DE LA ESTIMULACIÓN VAGAL}

Además de la eficacia sobre la reducción del número de crisis se han aportado múltiples datos sobre otros indicadores clínicos. Pacientes y familiares han confirmadounbeneficioen otras áreas, como el nivel de alerta, la memoria, el estado de ánimo y la expresión verbal. La tranquilidad que confiere el hecho de poder detener la crisis es un factor muy apreciado [77,78]. La reducción de la dosis y el número de los fármacos antiepilépticos contribuye también a la mejoría global, a la disminución de los posibles efectos secundarios y a mejorar la alerta [79]. Llama la atención que un paciente con síndrome de Unverricht-Lundborg mejoró no sólo el control de las crisis (en más del90\%), sino también la sintomatologíacerebelosa [80].

\section{EFECTOS SECUNDARIOS Y COMPLICACIONES}

Esta técnica puede tener complicaciones derivadas del procedimiento quirúrgico o bien de la presencia de un cuerpo extraño. Otras provienen de la actividad propia del sistema, debido al depósito de materiales tóxicos o a la excitación eléctrica inducida en el nervio vago o en territorios vecinos [81].

La operación quirúrgica del implante se considera como una cirugía de bajo riesgo; pueden ocurrir hemorragias o infecciones, de modo poco frecuente como en otras cirugías en el área cervical superficial [82]. Un paciente en el estudio sufrió una parálisis de la cuerda vocal izquierda que persistía tras un año de seguimiento [47].

Las quejas más frecuentes son: ronquera, tos, dolor cervical, disfonía y alteración del tono de voz. La disfonía y la sensación anormal en la faringe es más frecuente y molesta si la estimulación se lleva a cabo con parámetros de estimulación alta. Se ha documentado la aducción forzada de una cuerda vocal [83]. Resultan menos frecuentes la disnea y el hipo. En niños con retraso psicomotor se ha descrito dificultad para la deglución [84]. También hay, ocasionalmente, cefalea o náuseas.

Entre los nervios vecinos que pueden verse alterados de algún modo se han comunicado el plexo simpático cervical [85], el nervio frénico [86] y el glosofaríngeo [87], además de la presentación de tortícolis debido a la inducción de un espasmo en el esternocleidomastoideo por afectación del núcleo espinal [88].

Una de las complicaciones más graves es la alteración del ritmo cardíaco. La colocación del electrodo en el vagoizquierdo intenta evitar esta complicación pero, a pesar de ello, ha habido casos de bradicardia e incluso asistolia [89-91]. Tatum et al refieren haber tenido cuatro casos de asistolia en quirófano durante la prueba inicial de estimulación del estimulador vagal $\mathrm{y}$, por ello, recomiendan que las pruebas de estimulación posteriores se realicen con control continuo de electrocardiograma [92]. Lesser también refiere esta posibilidad [90]. Otros autores inciden en la ausencia de cambios en la frecuencia cardíaca con la estimulación [93].

Los pacientes con enfermedad pulmonar obstructiva crónica (EPOC) o con apneas del sueño deben vigilarse con detenimiento por la posibilidad de empeorar la función respiratoria o incrementarelíndice de apneas $[94,95]$. Se ha descrito una mayor frecuencia de muerte súbita no explicada en pacientes epilépticos a los que se implantó el estimulador. La explicación de este hallazgo reside probablemente en la selección de los pacientes, ya que aquellos pacientes que poseen estimulador del nervio vago constituyen un grupo con epilepsias farmacorresistentes, mayor frecuencia basal de crisis y, en consecuencia, mayor riesgo de muerte súbita [96]. Respecto a la sintomatología psiquiátrica, aunque lo habitual es un efecto positivo, hubo un paciente que presentó un cuadro de psicosis aguda [97].

Los problemas técnicos son poco frecuentes. En el caso de objetivarse un aumento de resistencia del electrodo, el problema provoca un mayor gasto de batería. En algún caso aislado se ha presentado un cortocircuito en el sistema que produjo una estimulación directa, o problemas en la programación del generador [98]. 
Durante los estudios, un paciente sufrió un infarto agudo de miocardio [99] y a otro se le diagnosticó una púrpura trombocitopénica [52], trastornos probablemente no relacionados con la estimulación vagal.

Globalmente, estas complicaciones son poco frecuentes e importantes, y suelen disminuir al ajustar los parámetros de estimulación. Un dato que confirma esta afirmación es el alto porcentaje de pacientes que llegan a completar los estudios correspondientes $(96,7 \%$ tras un año, $84,7 \%$ tras dos años y $72,1 \%$ después de tres años [57].

\section{CONCLUSIONES}

Los resultados de los ensayos y la experiencia acumulada en los últimos años sitúan el estimulador vagal como una posibilidad más dentro del tratamiento de la epilepsia. Aunque es un sistema caro, la disminución de crisis y destatus epilepticushace bajar muy significativamente los cuidados médicos. Por eso, aunque estos pacientes deben continuar con fármacos antiepilépticos, la relación coste/beneficio parece positiva [100]. El número de pacientes a los que se ha implantado un estimulador ha aumentado de modo exponencial desde 1997.

En Estados Unidos se ha aprobado para uso en epilepsias farmacorresistentes no susceptibles de tratamientoquirúrgico.Se considera como un tratamiento paliativo que trata de disminuir la frecuencia de las crisis epilépticas en pacientes muy incapacitados [101]. Los datos de los ensayos controlados son de adultos con epilepsias focales, pero parece posible que puedabeneficiar a otros grupos de pacientes. Su eficacia es comparable, oincluso mayor, a la de cada uno de los nuevos fármacos antiepilépticos $[46,102]$. El comité de evaluación de la estimulación vagal opinó que el estimulador vagal se indica en adultos y niños mayores de 12 años con epilepsia farmacorresistente y no candidatos a trata- miento curativo mediante cirugía resectiva [47]. El comité manifestóla conveniencia de descartar la presencia de crisis de origen noepiléptico mediante una monitorización con vídeo-EEGantes de laimplantación del sistema. Dado que el estimulador del nervio vago raramente produce un control completo de las crisis y, de algún modo, constituye un tratamiento invasivo, se recomienda únicamente en pacientes en los cuales los fármacos no consiguen reducir el alto número de crisis o producen efectos secundarios importantes, afectando gravemente a su calidad de vida. Los tipos de epilepsia de estos pacientes suelen serfocales o secundariamente generalizadas. En las crisis focales con síntomas iniciales, el estimulador puede indicarse especialmente por la posibilidad de intentar abortar la crisis en su inicio.

Uno de los puntos no evaluados es la repercusión en cada uno de los tipos de crisis y síndromes epilépticos. Determinar los factores pronósticos para la respuesta de cada uno de estos tipos es importante porque muchos pacientes epilépticos están limitados para su vida cotidiana por la presencia de un tipo concreto de crisis más que por el número global de crisis, debido a su horario, a su duración, a que las crisis se acompañen de caídas, a que ocurran durante la vigilia, etc. Muchas veces, el control de esas crisis puede resultar más relevante que la disminución total en el número de episodios críticos.

En los próximos años debe seguirse investigando para conocer su mecanismo de acción. Además, será necesario perfeccionar los aspectos técnicos del aparato, determinar los parámetros óptimos de estímulo, disminuir los efectos secundarios y los costes y encontrar nuevas aplicaciones. La utilización en grupos más amplios de pacientes será el examen definitivo, ya que se conocerá con exactitud su eficacia y su seguridad y ayudaráa determinar quiénes son los candidatos idóneos para recibir este tratamiento.

\section{BIBLIOGRAFÍA}

1. Spencer DD, Spencer SS. Surgery for epilepsy. Neurol Clin 1985; 3: 313-30.

2. Engel J. Surgery for seizures. N Engl J Med 1996; 334: 647-52.

3. Uthman BM, Wilder BJ, Penry JK, Dean C, Ramsay RE, Reid SA, et al. Treatment of epilepsy by stimulation of the vagus nerve. Neurology 1993; 43: 1338-45.

4. Wright GD, McLellan DL, Brice JG. A double-blind trial of chronic cerebellar stimulation in twelve patients with severe epilepsy. J Neurol Neurosurg Psychiatry 1984; 47: 769-74.

5. Fisher RS, Uematsu S, Krauss GL, Cysyk BJ, McPherson R, Lesser $\mathrm{RP}$, et al. Placebo-controlled pilot study of centromedian thalamic stimulation in treatment of intractable seizures. Epilepsia 1992; 33: 841-51.

6. Velasco F, Velasco M, Jiménez F, Velasco AL, Brito F, Rise M, et al. Predictors in the treatment of difficult-to-control seizures by electrical stimulation of the centromedian thalamic nucleus. Neurosurgery 2000; 47: 295-304.

7. Velasco F, Velasco M, Velasco AL, Jiménez F, Márquez I, Rise M. Electrical stimulation of the centromedian thalamic nucleus in control of seizures: long-term studies. Epilepsia 1995; 36: 63-71.

8. Lesser RP. Unexpected places: how did vagus nerve stimulation become a treatment for epilepsy? Neurology 1999; 52: 1117-8.

9. Ben-Menachem E. Modern management of epilepsy: vagus nerve stimulation. Baillieres Clin Neurol 1996; 5: 841-8.

10. Penry JK, Dean JC. Prevention of intractable partial seizures by intermittent vagal stimulation in humans: preliminary results. Epilepsia 1990; 31 (Suppl): S40-3.

11. Vagus nerve stimulation for the control of epilepsy. Proceedings of a symposium held in conjunction with the American Epilepsy Society annual meeting. Boston, Massachusetts, December 2, 1989. Epilepsia 1990; 31 (Suppl): S1-60.

12. Schachter SC, Saper CB. Vagus nerve stimulation. Epilepsia 1998; 39:677-86.

13. Álvarez LA, Dean P, Jayakar P, Duchowny M, Resnick T, Dunoyer
C, et al. Tratamiento de la epilepsia por medio de la estimulación vagal. Rev Neurol 1999; 29: 385-7.

14. Terry R, Tarver WB, Zabara J. An implantable neurocybernetic prosthesis system. Epilepsia 1990; 31 (Suppl): S33-7.

15. Reid SA. Surgical technique for implantation of the neurocybernetic prosthesis. Epilepsia 1990; 31 (Suppl): S38-9.

16. Espinosa J, Aiello MT, Naritoku DK. Revision and removal of stimulating electrodes following long-term therapy with the vagus nerve stimulator. Surg Neurol 1999; 51: 659-64.

17. [http://www.cyberonics.com/physician/dyathermy-clinicians-us.htm] 27.08.2001.

18. Maniker A, Liu WC, Marks D, Moser K, Kalnin A. Positioning of vagal nerve stimulators: technical note. Surg Neurol 2000; 53: 178-81.

19. Zagon A, Kemeny AA. Slow hyperpolarization in cortical neurons: a possible mechanism behind vagus nerve simulation therapy for refractory epilepsy? Epilepsia 2000; 41: 1382-9.

20. Naritoku DK, Terry WJ, Helfert RH. Regional induction of fos immunoreactivity in the brain by anticonvulsant stimulation of the vagus nerve. Epilepsy Res 1995; 22: 53-62.

21. Krahl SE, Clark KB, Smith DC, Browning RA. Locus coeruleus lesions suppress the seizure-attenuating effects of vagus nerve stimulation. Epilepsia 1998; 39: 709-14.

22. McLachlan RS. Suppression of interictal spikes and seizures by stimulation of the vagus nerve. Epilepsia 1993; 34: 918-23.

23. Zabara J. Inhibition of experimental seizures in canines by repetitive vagal stimulation. Epilepsia 1992; 33: 1005-12.

24. Woodbury JW, Woodbury DM. Vagal stimulation reduces the severity of maximal electroshock seizures in intact rats: use of a cuff electrode for stimulating and recording. Pacing Clin Electrophysiol 1991; 14:94-107.

25. Fanselow EE, Reid AP, Nicolelis MA. Reduction of pentylenetetrazole-induced seizure activity in awake rats by seizure-triggered trigeminal nerve stimulation. J Neurosci 2000; 20: 8160-8.

26. Krahl SE, Senanayake SS, Handforth A. Seizure suppression by sys- 
temic epinephrine is mediated by the vagus nerve. Epilepsy Res 2000; 38: 171-5.

27. Rutecki P. Anatomical, physiological, and theoretical basis for the antiepileptic effect of vagus nerve stimulation. Epilepsia 1990; 31 (Suppl): S1-6.

28. Fernández GA, Martínez A, Valdés CA, Magdaleno MV, Martínez D, Fernández MR. Vagus nerve prolonged stimulation in cats: effects on epileptogenesis (amygdala electrical kindling): behavioral and electrographic changes. Epilepsia 1999; 40: 822-9.

29. Olejniczak PW, Fisch BJ, Carey M, Butterbaugh G, Happel L, Tardo C. The effect of vagus nerve stimulation on epileptiform activity recorded from hippocampal depth electrodes. Epilepsia 2001; 42: 423-9.

30. Hammond EJ, Uthman BM, Reid SA, Wilder BJ. Electrophysiological studies of cervical vagus nerve stimulation in humans. I. EEG effects. Epilepsia 1992; 33: 1013-20.

31. Salinsky MC, Burchiel KJ. Vagus nerve stimulation has no effect on awake EEG rhythms in humans. Epilepsia 1993; 34: 299-304.

32. Hammond EJ, Uthman BM, Reid SA, Wilder BJ, Ramsay RE. Vagus nerve stimulation in humans: neurophysiological studies and electrophysiological monitoring. Epilepsia 1990; 31 (Suppl): S51-9.

33. Lockard JS, Congdon WC, DuCharme LL. Feasibility and safety of vagal stimulation in monkey model. Epilepsia 1990; 31 (Suppl): S20-6.

34. Dasheiff RM, Sandberg T, Thompson J, Arrambide S. Vagal nerve stimulation does not unkindle seizures. J Clin Neurophysiol 2001; 18 : 68-74.

35. Hammond EJ, Uthman BM, Reid SA, Wilder BJ. Electrophysiologic studies of cervical vagus nerve stimulation in humans. II. Evoked potentials. Epilepsia 1992; 33: 1021-8.

36. Naritoku DK, Morales A, Pencek TL, Winkler D. Chronic vagus nerve stimulation increases the latency of the thalamocortical somatosensory evoked potential. Pacing Clin Electrophysiol 1992; 15: 1572-8.

37. Ko D, Heck C, Grafton S, Apuzzo ML, Couldwell WT, Chen T, et al. Vagus nerve stimulation activates central nervous system structures in epileptic patients during PET H2(15)O blood flow imaging. Neurosurgery 1996; 39: 426-30.

38. Labar D, Nikolov B, Tarver B, Fraser R. Vagus nerve stimulation for symptomatic generalized epilepsy: a pilot study. Epilepsia 1998; 39: 201-5.

39. Henry TR, Bakay RA, Votaw JR, Pennell PB, Epstein CM, Faber TL, et al. Brain blood flow alterations induced by therapeutic vagus nerve stimulation in partial epilepsy. I. Acute effects at high and low levels of stimulation. Epilepsia 1998; 39: 983-90.

40. Henry TR, Votaw JR, Pennell PB, Epstein CM, Bakay RA, Faber TL, et al. Acute blood flow changes and efficacy of vagus nerve stimulation in partial epilepsy. Neurology 1999; 52: 1166-73.

41. Ring HA, White S, Costa DC, Pottinger R, Dick JP, Koeze T, et al. A SPECT study of the effect of vagal nerve stimulation on thalamic activity in patients with epilepsy. Seizure 2000; 9: 380-4.

42. Vonck K, Boon P, Van-Laere K, D'Have M, Vandekerckhove T, O'Connor S, et al. Acute single photon emission computed tomographic study of vagus nerve stimulation in refractory epilepsy. Epilepsia 2000; 41: 601-9

43. Ben-Menachem E, Hamberger A, Hedner T, Hammond EJ, Uthman BM, Slater J, et al. Effects of vagus nerve stimulation on amino acids and other metabolites in the CSF of patients with partial seizures. Epilepsy Res 1995; 20: 221-7.

44. Woodbury DM, Woodbury JW. Effects of vagal stimulation on experimentally induced seizures in rats. Epilepsia 1990; 31 (Suppl): S7-19.

45. Landy HJ, Ramsay RE, Slater J, Casiano RR, Morgan R. Vagus nerve stimulation for complex partial seizures: surgical technique, safety, and efficacy. J Neurosurg 1993; 78: 26-31.

46. Fisher RS, Krauss GL, Ramsay E, Laxer K, Gates J. Assessment of vagus nerve stimulation for epilepsy: report of the Therapeutics and Technology Assessment Subcommittee of the American Academy of Neurology. Neurology 1997; 49: 293-7.

47. Fisher RS, Handforth A. Reassessment of vagus nerve stimulation for epilepsy: a report of the Therapeutics and Technology Assessment Subcommittee of the American Academy of Neurology. Neurology 1999; 53: 666-9.

48. Ben-Menachem E, Manon ER, Ristanovic R, Wilder BJ, Stefan H, Mirza W, et al. Vagus nerve stimulation for treatment of partial seizures. 1. A controlled study of effect on seizures. First International Vagus Nerve Stimulation Study Group. Epilepsia 1994; 35: 616-26.

49. Anonymous. A randomized controlled trial of chronic vagus nerve stimulation for treatment of medically intractable seizures. The Vagus Nerve Stimulation Study Group. Neurology 1995; 45: 224-30.

50. Labar D, Murphy J, Tecoma E. Vagus nerve stimulation for medicationresistant generalized epilepsy. E04 VNS Study Group. Neurology 1999; 52: 1510-2.
51. Handforth A, DeGiorgio CM, Schachter SC, Uthman BM, Naritoku DK, Tecoma ES, et al. Vagus nerve stimulation therapy for partial-onset seizures: a randomized active-control trial. Neurology 1998; 51: 48-55.

52. George R, Salinsky M, Kuzniecky R, Rosenfeld W, Bergen D, Tarver WB, et al. Vagus nerve stimulation for treatment of partial seizures. 3. Long-term follow-up on first 67 patients exiting a controlled study. First International Vagus Nerve Stimulation Study Group. Epilepsia 1994; 35: 637-43

53. Salinsky MC, Uthman BM, Ristanovic RK, Wernicke JF, Tarver WB. Vagus nerve stimulation for the treatment of medically intractable seizures. Results of a 1-year open-extension trial. Vagus Nerve Stimulation Study Group. Arch Neurol 1996; 53: 1176-80.

54. DeGiorgio CM, Schachter SC, Handforth A, Salinsky M, Thompson J, Uthman B, et al. Prospective long-term study of vagus nerve stimulation for the treatment of refractory seizures. Epilepsia 2000; 41: 1195-200.

55. DeGiorgio CM, Thompson J, Lewis P, Arrambide S, Naritoku D, Handforth A, et al. Vagus nerve stimulation: analysis of device parameters in 154 patients during the long-term XE5 study. Epilepsia 2001; 42: 1017-20.

56. Amar AP, DeGiorgio CM, Tarver WB, Apuzzo ML. Long-term multicenter experience with vagus nerve stimulation for intractable partial seizures: results of the XE5 trial. Stereotact Funct Neurosurg 1999; 73: 104-8.

57. Morris GL, Mueller WM. Long-term treatment with vagus nerve stimulation in patients with refractory epilepsy. The Vagus Nerve Stimulation Study Group E01-E05. Neurology 1999; 53: 1731-5.

58. Labar D. Vagus nerve stimulation for intractable epilepsy in children. Dev Med Child Neurol 2000; 42: 496-9.

59. Wakai S, Kotagal P. Vagus nerve stimulation for children and adolescents with intractable epilepsies. Pediatr Int 2001; 43: 61-5.

60. Farooqui S, Boswell W, Hemphill JM, Pearlman E. Vagus nerve stimulation in pediatric patients with intractable epilepsy: case series and operative technique. Am Surg 2001; 67: 119-21.

61. Crumrine PK. Vagal nerve stimulation in children. Semin Pediatr Neurol 2000; 7: 216-23.

62. Hornig GW, Murphy JV, Schallert G, Tilton C. Left vagus nerve stimulation in children with refractory epilepsy: an update. South Med J 1997; 90: 484-8.

63. Lundgren J, Amark P, Blennow G, Stromblad LG, Wallstedt L. Vagus nerve stimulation in 16 children with refractory epilepsy. Epilepsia 1998; 39: 809-13.

64. Hosain S, Nikalov B, Harden C, Li M, Fraser R, Labar D. Vagus nerve stimulation treatment for Lennox-Gastaut syndrome. J Child Neurol 2000; 15: 509-12.

65. Murphy JV. Left vagal nerve stimulation in children with medically refractory epilepsy. The Pediatric VNS Study Group. J Pediatr 1999; 134: 563-6.

66. Patwardhan RV, Stong B, Bebin EM, Mathisen J, Grabb PA. Efficacy of vagal nerve stimulation in children with medically refractory epilepsy. Neurosurgery 2000; 47: 1353-7.

67. Murphy JV, Wheless JW, Schmoll CM. Left vagal nerve stimulation in six patients with hypothalamic hamartomas. Pediatr Neurol 2000; 23: $167-8$.

68. Ben-Menachem E, Hellstrom K, Waldton C, Augustinsson LE. Evaluation of refractory epilepsy treated with vagus nerve stimulation for up to 5 years. Neurology $1999 ; 52: 1265-7$.

69. Amar AP, Heck CN, Levy ML, Smith T, DeGiorgio CM, Oviedo S, et al. An institutional experience with cervical vagus nerve trunk stimulation for medically refractory epilepsy: rationale, technique, and outcome. Neurosurgery 1998; 43: 1265-76.

70. Alsaadi TM, Laxer KD, Barbaro NM, Marks WJ, García PA. Vagus nerve stimulation for the treatment of bilateral independent temporal lobe epilepsy. Epilepsia 2001; 42: 954-6.

71. Rafael H, Moromizato P. Vagus nerve stimulation (VNS) may be useful in treating patients with symptomatic generalized epilepsy. Epilepsia 1998; 39: 1018 .

72. Michael JE, Wegener K, Barnes DW. Vagus nerve stimulation for intractable seizures: one year follow-up. J Neurosci Nurs 1993; 25: 362-6.

73. Morrow JI, Bingham E, Craig JJ, Gray WJ. Vagal nerve stimulation in patients with refractory epilepsy. Effect on seizure frequency, severity and quality of life. Seizure 2000; 9: 442-5.

74. Sirven JI, Sperling M, Naritoku D, Schachter S, Labar D, Holmes M, et al. Vagus nerve stimulation therapy for epilepsy in older adults. Neurology 2000; 54: 1179-82.

75. Parker AP, Polkey CE, Binnie CD, Madigan C, Ferrie CD, Robinson RO. Vagal nerve stimulation in epileptic encephalopathies. Pediatrics 1999; 103: 778-82. 
76. Koutroumanidis M, Hennessy MJ, Binnie CD, Polkey CE. Aggravation of partial epilepsy and emergence of new seizure type during treatment with VNS. Neurology 2000; 55: 892-3.

77. Sackeim HA, Keilp JG, Rush AJ, George MS, Marangell LB, Dormer JS, et al. The effects of vagus nerve stimulation on cognitive performance in patients with treatment-resistant depression. Neuropsychiatry Neuropsychol Behav Neurol 2001; 14: 53-62.

78. Elger G, Hoppe C, Falkai P, Rush AJ, Elger CE. Vagus nerve stimulation is associated with mood improvements in epilepsy patients. Epilepsy Res 2000; 42: 203-10.

79. Tatum WO, Johnson KD, Goff S, Ferreira JA, Vale FL. Vagus nerve stimulation and drug reduction. Neurology 2001; 56: 561-3.

80. Smith B, Shatz R, Elisevich K, Bespalova IN, Burmeister M. Effects of vagus nerve stimulation on progressive myoclonus epilepsy of Unverricht-Lundborg type. Epilepsia 2000; 41: 1046-8.

81. Agnew WF, McCreery DB. Considerations for safety with chronically implanted nerve electrodes. Epilepsia 1990; 31 (Suppl): S27-32.

82. Ortler M, Luef G, Kofler A, Bauer G, Twerdy K. Deep wound infection after vagus nerve stimulator implantation: treatment without removal of the device. Epilepsia 2001; 42: 133-5.

83. Zumsteg D, Jenny D, Wieser HG. Vocal cord adduction during vagus nerve stimulation for treatment of epilepsy. Neurology 2000; 54 : 1388-9.

84. Lundgren J, Ekberg O, Olsson R. Aspiration: a potential complication to vagus nerve stimulation. Epilepsia 1998; 39: 998-1000.

85. Kim W, Clancy RR, Liu GT. Horner syndrome associated with implantation of a vagus nerve stimulator. Am J Ophthalmol 2001; 131 : 383-4.

86. Leijten FS, Van-Rijen PC. Stimulation of the phrenic nerve as a complication of vagus nerve pacing in a patient with epilepsy. Neurology 1998; 51: 1224-5.

87. Duhaime A, Melamed M, Clancy R. Tonsillar pain mimicking glossopharyngeal neuralgia as a complication of vagus nerve stimulation: case report. Epilepsia 2000; 41: 903-5.

88. Iriarte J, Lázaro D, Viteri J, Artieda J. Tetanización del esternocleidomastoideo inducido por estimulador vagal. Neurologiía 2000; 15 : 556.

89. Asconapé J, Moore D, Zipes D, Hartman L, Duffell WH. Bradycardia and asystole with the use of vagus nerve stimulation for the treatment

\section{ESTIMULACIÓN VAGAL}

\section{EN EL TRATAMIENTO DE LA EPILEPSIA}

Resumen. Introducción. La estimulación cerebral mediante estímulos eléctricos aplicados en el nervio vago a través de un generador implantado subcutáneamente es un tratamiento de la epilepsia introducido en los últimos años en la práctica médica habitual. Desarrollo. En Estados Unidos, la FDA lo aprobó para uso en pacientes con epilepsias farmacorresistentes en 1997. Su mecanismo de acción no se conoce. En la actualidad se considera como un tratamiento paliativo, con un porcentaje de pacientes que experimentan una mejoría significativa alrededor del 50\%. En pacientes con epilepsias especialmente difíciles, rebeldes a los fármacos y no candidatos a cirugía, este recurso técnico parece hoy en día una opción razonable. [REV NEUROL 2002; 34: 511-8]

Palabras clave. Epilepsia farmacorresistente. Estimulador vagal. of epilepsy: a rare complication of intraoperative device testing. Epilepsia 1999; 40: 1452-4.

90. Lesser RP. Ventricular asystole during vagus nerve stimulation for epilepsy in humans. Neurology 2000; 54: 776.

91. Lanska DJ. Ventricular asystole during vagus nerve stimulation for epilepsy in humans. Neurology 2000; 54: 775.

92. Tatum WO, Moore DB, Stecker MM, Baltuch GH, French JA, Ferreira JA, et al. Ventricular asystole during vagus nerve stimulation for epilepsy in humans. Neurology 1999; 52: 1267-9.

93. Setty AB, Vaughn BV, Quint SR, Robertson KR, Messenheimer JA. Heart period variability during vagal nerve stimulation. Seizure 1998; 7: 213-7.

94. Lotvall J, Lunde H, Augustinsson LE, Hedner T, Svedmyr N, BenMenachem E. Airway effects of direct left-sided cervical vagal stimulation in patients with complex partial seizures. Epilepsy Res 1994; 18: 149-54.

95. Malow BA, Edwards J, Marzec M, Sagher O, Fromes G. Effects of vagus nerve stimulation on respiration during sleep: a pilot study. Neurology 2000; 55: 1450-4.

96. Annegers JF, Coan SP, Hauser WA, Leestma J, Duffell W, Tarver B. Epilepsy, vagal nerve stimulation by the NCP system, mortality, and sudden, unexpected, unexplained death. Epilepsia 1998; 39: 206-12.

97. Gatzonis SD, Stamboulis E, Siafakas, Angelopoulos E, Georgaculias N, Sigounas E, et al. Acute psychosis and EEG normalisation after vagus nerve stimulation. J Neurol Neurosurg Psychiatry 2000; 69: 278-79.

98. Ramsay RE, Uthman BM, Augustinsson LE, Upton AR, Naritoku D, Willis J, et al. Vagus nerve stimulation for treatment of partial seizures. 2. Safety, side effects, and tolerability. First International Vagus Nerve Stimulation Study Group. Epilepsia 1994; 35: 627-36.

99. A randomized controlled trial of chronic vagus nerve stimulation for treatment of medically intractable seizures. The Vagus Nerve Stimulation Study Group. Neurology 1995; 45: 224-30.

100. Boon P, Vonck K, D'Have M, O'Connor S, Vandekerckhove T, De-Reuck J. Cost-benefit of vagus nerve stimulation for refractory epilepsy. Acta Neurol Belg 1999; 99: 275-80.

101. Chadwick D. Vagal-nerve stimulation for epilepsy. Lancet 2001; 357: 1726-7.

102. McLachlan RS. Vagus nerve stimulation for intractable epilepsy: a review. J Clin Neurophysiol 1997; 14: 358-68.

\section{ESTIMULAÇÃO VAGAL}

\section{NO TRATAMENTO DA EPILEPSIA}

Resumo. Introdução. A estimulação cerebral por estímulos eléctricos aplicados no nervo vago através de um gerador implantado subcutaneamente é um tratamento da epilepsia introduzido nos últimos anos na prática médica habitual. Desenvolvimento. Nos Estados Unidos, a FDA aprovou-o para utilização em doentes farmaco-resistentes em 1997. O seu mecanismo de acção não é conhecido. Presentemente é considerado um tratamento paliativo, com uma percentagem de doentes que experimentam melhoria significativa de cerca de 50\%. Em doentes com epilepsias particularmente difíceis, refractários aos fármacos e não candidatos a cirurgia, este recurso técnico parece hoje em dia uma opção razoável. [REV NEUROL 2002; 34: 511-8]

Palavras chave. Epilepsia farmaco-resistente. Estimulador vagal. 